\title{
Produção de geopolímeros à base de metacaulim e cerâmica vermelha
}

\section{(Metakaolin and red ceramic based geopolymers production)}

\author{
A. G. de S. Azevedo ${ }^{I}$ K. Strecker ${ }^{l}, C$. T. Lombardi ${ }^{I}$ \\ ${ }^{1}$ Universidade Federal de São João del-Rei, Pç. Frei Orlando 170, 36307-352, São João del-Rei, MG, Brasil
}

\begin{abstract}
Resumo
O aumento de $\mathrm{CO}_{2}$ gerado durante a fabricação e aplicação do cimento Portland tem gerado a necessidade de se estudar a utilização de um novo sistema de ligantes. Os materiais provenientes da ativação alcalina de aluminossilicatos apresentam propriedades similares àquelas observadas no cimento Portland, sendo possível utilizá-los em algumas aplicações da construção civil. Nesse trabalho, investigou-se a produção de geopolímeros fabricados com metacaulim e cerâmica vermelha. Os materiais apresentaram resistência mecânica próxima de $30 \mathrm{MPa}$ após 28 dias de cura. Os resultados acerca da microestrutura dos ligantes inorgânicos constaram que a utilização da cerâmica vermelha não modificou as reações de geopolimerização. Entretanto, a presença da cerâmica vermelha modificou os valores de absorção de água, porosidade aparente e densidade. A superfície apresentou algumas partículas de cerâmica vermelha que não participaram da reação. A baixa taxa de reação da cerâmica vermelha foi relacionada com as alterações observadas no ligante álcali-ativado.
\end{abstract}

Palavras-chave: geopolímeros, metacaulim, cerâmica vermelha, geopolimerização.

\begin{abstract}
The increase of $\mathrm{CO}_{2}$ released during the application and production of Portland cement has promoted the necessity to study a new kind of binder. The alkali-activated materials have similar properties of Portland cement; this way, some applications in the civil construction can be made using these materials. The aim of this work was to produce metakaolin and red ceramic based geopolymers. The specimens showed mechanical strength close to $30 \mathrm{MPa}$ after 28 days of curing. The analysis of the microstructure demonstrated that the use of red ceramic did not modify the geopolymerization reactions. However, the red ceramic promoted changes in the water absorption, porosity and density values. The geopolymers surface showed some intact red ceramic particles. This was related to low reactivity of the raw material that promoted the observed modifications of the geopolymeric specimens.
\end{abstract}

Keywords: geopolymers, metakaolin, red ceramic, geopolymerization.

\section{INTRODUÇÃO}

Nas últimas décadas houve um crescente aumento tecnológico relacionado com pesquisas sobre novos materiais. Esses novos materiais devem ser produzidos com melhorias em suas aplicações e, se possível, com uma preocupação em relação ao impacto ambiental causado por eles. A indústria da construção civil está dentre os setores que mais degradam o meio ambiente durante a produção dos materiais necessários para implantação $[1,2]$. O cimento Portland (CP) é um dos materiais mais utilizados na indústria da construção civil e sua fabricação promove a liberação de elevadas quantidades de $\mathrm{CO}_{2}$ para a atmosfera e consome grande quantidade de energia durante a produção do clínquer [3]. De acordo com o relatório produzido em 2013 pelo Sindicato Nacional da Indústria do Cimento, no mesmo ano, o Brasil consumiu cerca de 70000 toneladas de cimento Portland [3]. Devido ao grande consumo desse material e com sua impactante destruição ambiental um novo ligante capaz de competir com o CP como ligante na construção civil se faz necessário [4-6].

Uma forma de minimizar esse impacto ambiental é a utilização de materiais álcali-ativados [4]. Esses materiais, também conhecidos como geopolímeros ou polímeros inorgânicos são materiais ricos em aluminossilicatos, apresentando estruturas que variam de acordo com a composição química: poli(sialato), -Si-O-Al-O- (razão $\mathrm{Si}: \mathrm{Al}=1: 1) ; \quad$ poli(sialato-siloxo), $\quad$-Si-O-Al-O-Si-O( $\mathrm{Si}: \mathrm{Al}=2: 1$ ) e poli(sialato-dissiloxo),-Si-O-Al-O-Si-O-Si-O( $\mathrm{Si}: \mathrm{Al}=3: 1$ ) [6-8]. Esses polímeros inorgânicos apresentam grandes vantagens quando comparados ao $\mathrm{CP}$. Entre as várias propriedades podemos destacar a elevada resistência mecânica, ganho de resistência mecânica em curtos períodos de cura, resistência a ataques ácidos e de sulfatos, resistência a ciclos de gelo-degelo, estabilidade estrutural quando submetidos a elevadas temperaturas, entre outros [5, 9-16]. O termo geopolímero foi primeiramente utilizado pelo francês Davidovits na década de 1970 e o processo se assemelha ao mecanismo de obtenção dos polímeros orgânicos [17, 18]. A composição mineralógica do material de partida, temperatura e tempo de cura, quantidade de água e a concentração dos compostos presentes na solução alcalina ativadora são os principais parâmetros controlados durante a produção desses materiais [19-23]. A obtenção desse material pode ser feita por meio da ativação alcalina de precursores ricos e aluminossilicatos, tais como metacaulim, 
cinza volante e escória granulada de alto forno $[5,15,19,24$ 32]. A alteração do material de partida promove alterações nas reações e, consequentemente, nas propriedades finais do ligante após o enrijecimento [5].

Esse trabalho apresenta a produção de geopolímeros à base de metacaulim (MK) com a substituição parcial do MK por cerâmica vermelha (CV). A escolha da cerâmica vermelha se deu principalmente por se tratar de um material sem grande reaproveitamento após sua aplicação na construção civil. A produção de geopolímeros à base de metacaulim e CV é interessante uma vez que contribui para a reutilização desse material pouco reciclado, favorecendo a diminuição dessa classe de resíduos em aterros destinados a materiais de demolição da indústria da construção civil.

\section{MATERIAIS E MÉTODOS}

Materiais: silicato de sódio $\left(\mathrm{Na}_{2} \mathrm{SiO}_{3}\right)$ : foi adquirido da Diatom (R-2252) e apresentou a composição demonstrada na Tabela I; hidróxido de sódio $(\mathrm{NaOH})$ : as soluções alcalinas foram preparadas com hidróxido de sódio em escamas do tipo P.A. da Sulfal Química (pureza de 98\%); metacaulim $(M K)$ : foi proveniente da Metacaulim do Brasil; cerâmica vermelha $(C V)$ : foi obtida da Pó Piacentini (pó de telha).

Métodos. Solução ativadora: para a ativação do metacaulim utilizou-se uma solução de $\mathrm{NaOH} 8 \mathrm{M}$. A solução foi preparada pela dissolução do hidróxido de sódio em água deionizada. O procedimento foi realizado $24 \mathrm{~h}$ antes da mistura com o MK e CV para se evitar modificações reacionais devido ao calor liberado pelo $\mathrm{NaOH} \mathrm{em} \mathrm{H}_{2} \mathrm{O}$. Da solução $8 \mathrm{M}$ foram substituídos $50 \%$ em massa com silicato de sódio alcalino $\left(\mathrm{Na}_{2} \mathrm{SiO}_{3}\right)$. Produção dos geopolímeros: para a produção dos geopolímeros o MK foi misturado com a solução ativadora (SA) em temperatura ambiente em uma razão $\mathrm{SA} / \mathrm{MK}+\mathrm{CV}$ de 0,9 (GPMK). A CV foi adicionada substituindo $50 \%$ em massa do metacaulim inicialmente adicionado (GPMK-CV). Para as amostras de GPMK foi necessário adicionar uma quantidade de água extra para se garantir uma melhor trabalhabilidade da pasta produzida. A composição das diferentes misturas estudadas é apresentada na Tabela II. As pastas foram adicionadas a moldes plásticos com dimensões de 50 x $25 \mathrm{~mm}$ (altura x diâmetro) e seladas utilizando um filme plástico para evitar a perda de água por evaporação. Os moldes foram vibrados em mesa vibratória por $60 \mathrm{~s}$ para se remover as bolhas formadas durante o preenchimento dos moldes. Após 24 h o filme plástico foi removido e as amostras foram curadas, em temperatura laboratorial, por 3, 7, 14 e 28 dias.

Caracterização dos materiais precursores e geopolímeros à base de $M K$ e $C V$ : foram utilizados 5 corpos de prova para cada condição para as medidas de resistência à compressão e análises microestruturais. Estas análises foram feitas por meio da moagem e peneiramento de fragmentos dos corpos de prova fraturados durante a compressão uniaxial. As amostras foram caracterizadas por meio da compressão uniaxial como descrito na norma NBR 5739 [13], usando uma máquina universal da Shimadzu (AG-X Plus) e velocidade de ensaio de $2 \mathrm{~mm} / \mathrm{min}$. Os dados obtidos foram tratados por meio da utilização do software Trapezium X v.1.2.6 (Shimadzu). O MK e a CV foram caracterizados utilizando um equipamento da Shimadzu (Energy Dispersive X-ray Fluorescence Spectrometer, EDX 8000) para a obtenção da composição química. A granulometria da CV foi obtida utilizando um aparelho da Malvern (Mastersizer 2000, 0,02$2000 \mu \mathrm{m})$. As amostras foram dispersas em água deionizada e as condições de ensaio foram: agitação de $1500 \mathrm{rpm}$, tempo de ultrassom de $2,5 \mathrm{~min}$, obscuração entre 10 e $20 \%$ e tempo de dispersão de $5 \mathrm{~min}$. A caracterização das fases presentes no material no MK, CV e nas pastas álcali-ativadas foi realizada utilizando um difratômetro da Shimadzu (XRD6000 ), com voltagem de $30 \mathrm{kV}$, corrente de $30 \mathrm{~mA}$, radiação de $\mathrm{CuK} \alpha(\lambda=0,15462 \mathrm{~nm})$ com passo de $0,02^{\circ}$ e velocidade de varredura de $2 \% \mathrm{mim}$, entre 5 e $60^{\circ}$. A identificação das fases foi realizada por comparação dos picos de difração obtidos com os fornecidos pelos arquivos do ICPDS (Inorganic Crystal Structure Database). As análises espectroscópicas foram feitas em um espectroscópio da Perkim Elmer (Sprectrum 1000). As amostras foram analisadas na forma de pastilhas misturando-se as amostras com $\mathrm{KBr}$ de elevada pureza (grau espectroscópico) e aplicando uma carga de 10

Tabela I - Composição e propriedades físicas do silicato alcalino, fornecidas pelo fabricante.

[Table I - Composition and physical properties of alkaline silicate (data provided by the manufacturer).]

\begin{tabular}{ccccccc}
\hline & \multicolumn{2}{c}{ Composição } & & Sólidos & Umidade & $\begin{array}{c}\text { Densidade a 25 } \\
\mathrm{Na}_{2} \mathrm{O}\end{array}$ \\
$\mathrm{SiO}_{2} \mathrm{C}\left(\mathrm{g} / \mathrm{cm}^{3}\right)$ & $\begin{array}{c}\text { Viscosidade } \\
(\mathrm{cP})\end{array}$ \\
\hline $14,985 \%$ & $33,574 \%$ & 2,40 & $48,559 \%$ & $51,441 \%$ & 1,589 & 1,350 \\
\hline
\end{tabular}

Tabela II - Composição das misturas dos geopolímeros à base de MK e cerâmica vermelha. [Table II - Mixture composition of metakaolin and red ceramic based geopolymers.]

\begin{tabular}{cccccc}
\hline Amostra & $\begin{array}{c}\mathrm{NaOH} 8 \text { mol. } \mathrm{L}^{-1} \\
(\% \text { massa })\end{array}$ & $\begin{array}{c}\mathrm{Na}_{2} \mathrm{SiO}_{3} \\
(\% \text { massa })\end{array}$ & $\begin{array}{c}\mathrm{MK} \\
(\% \text { massa })\end{array}$ & $\begin{array}{c}\mathrm{CV} \\
(\% \text { massa })\end{array}$ & $\begin{array}{c}\mathrm{H}_{2} \mathrm{O} \text { extra } \\
(\mathrm{g})\end{array}$ \\
\hline GPMK & 50 & 50 & 100 & 0 & 130 \\
GPMK-CV & 50 & 50 & 50 & 50 & 0 \\
\hline
\end{tabular}


ton por $60 \mathrm{~s}$. As análises foram obtidas a partir da média de 16 ciclos de varredura, no número de onda de 400 a 4000 $\mathrm{cm}^{-1}$ e resolução de $4 \mathrm{~cm}^{-1}$. Para a análise da morfologia das amostras MK, $\mathrm{CV}$ e dos materiais álcali-ativados foi utilizado um microscópio eletrônico da Tescan (Vega SBU). As propriedades físicas dos geopolímeros foram obtidas utilizando a norma britânica BS EN ISO 10545-3 (1997).

\section{RESULTADOS E DISCUSSÃO}

\section{Caracterização do $M K$ e $C V$}

Distribuição granulométrica e composição química: de acordo com o fabricante o material apresentou distribuição granulométrica com D50= $44 \mu \mathrm{m}$ e foi passante em peneira de 325 mesh. A Tabela III apresenta os resultados da composição química do MK e da CV. É possível observar que o MK foi composto basicamente de silício e alumínio, o que é de grande importância na síntese de polímeros inorgânicos. A razão molar das espécies $\mathrm{SiO}_{2} / \mathrm{Al}_{2} \mathrm{O}_{3}$ foi próxima de 2,16. A cerâmica vermelha foi moída em moinho de bolas por 24 h. O material obtido apresentou um D50 próximo de $21 \mu \mathrm{m}$ (Fig. 1). A cerâmica vermelha apresentou $\mathrm{SiO}_{2}$ como sendo o principal constituinte do material. Menor concentração de $\mathrm{Al}_{2} \mathrm{O}_{3}$ foi observada na composição química da $\mathrm{CV}$. O material apresentou elevada concentração de $\mathrm{Fe}_{2} \mathrm{O}_{3}$, que foi o responsável pela coloração avermelhada observada. A razão molar $\mathrm{SiO}_{2} / \mathrm{Al}_{2} \mathrm{O}_{3}$ se apresentou próxima de 5,75.

Difração de raios $X(D R X)$ : os difratogramas da Fig. 2 apresentam as fases presentes no MK e na CV. É possível observar no difratograma referente ao $\mathrm{MK}$ a presença de picos correspondentes às fases de quartzo $\left(\mathrm{SiO}_{2}\right)$, caulinita $\left[\mathrm{Al}_{2} \mathrm{Si}_{2} \mathrm{O}_{5}(\mathrm{OH})_{4}\right]$ e muscovita $\left[\mathrm{KAl}_{2} \mathrm{Si}_{3} \mathrm{AlO}_{10}(\mathrm{OH}, \mathrm{F})_{2}\right]$. A banda larga entre 18 e $38^{\circ} 2 \theta$ (presente em ambos materiais) demonstra a presença de material com ordenamento atômico de curto alcance. A presença desses aluminossilicatos amorfos é de extrema importância durante a produção dos geopolímeros devido à elevada taxa de dissolução destes no ambiente altamente alcalino. No difratograma referente à cerâmica vermelha é possível observar picos relacionados às fases de quartzo $\left(\mathrm{SiO}_{2}\right)$, analcita $\left(\mathrm{NaAlSi}_{2} \mathrm{O}_{6} \cdot \mathrm{H}_{2} \mathrm{O}\right)$, muscovita e goethita $[\mathrm{FeO}(\mathrm{OH})]$, sendo esse último relacionado à presença de elevada concentração de ferro no material.

Espectroscopia no infravermelho com transformada de Fourier (IVTF): na Fig. 3 estão presentes os espectros de absorção no infravermelho das amostras de MK e CV. No espectro do MK, a banda existente próxima de $3621 \mathrm{~cm}^{-1}$ foi

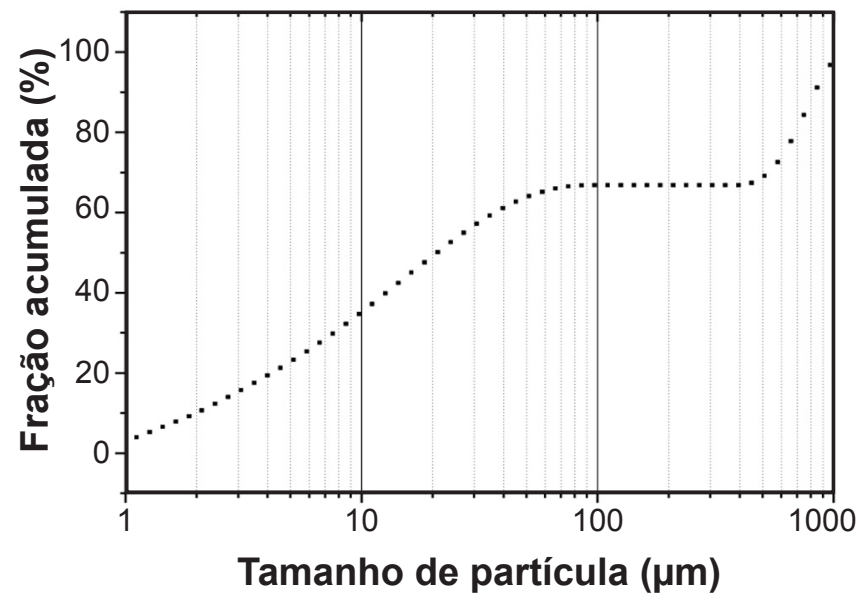

Figura 1: Curva de distribuição granulométrica da cerâmica vermelha utilizada na produção dos geopolímeros.

[Figure 1: Particle size distribution curve of red ceramic used to produce the geopolymers.]

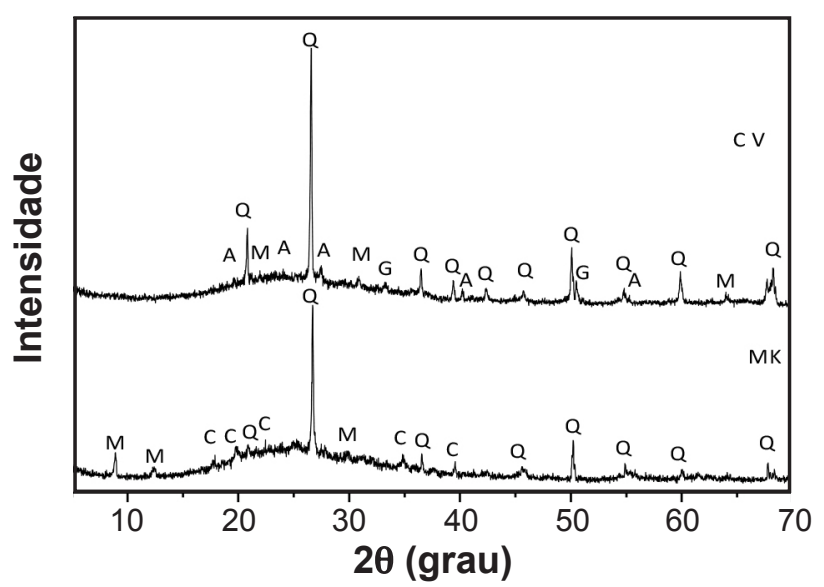

Figura 2: Difratogramas de raios X do metacaulim e da cerâmica vermelha $(\mathrm{CV})$ utilizados na produção dos geopolímeros. Q quartzo $\left(\mathrm{SiO}_{2}\right), \mathrm{M}$ - muscovita $\left[\mathrm{KAl}_{2} \mathrm{Si}_{3} \mathrm{AlO}_{10}(\mathrm{OH}, \mathrm{F})_{2}\right]$, A - analcita $\left(\mathrm{NaAlSi}{ }_{2} \mathrm{O}_{6} \cdot \mathrm{H}_{2} \mathrm{O}\right), \mathrm{C}$ - caulinita $\left[\mathrm{Al}_{2} \mathrm{Si}_{2} \mathrm{O}_{5}(\mathrm{OH})_{4}\right]$ e $\mathrm{G}$ - goethita $[\mathrm{FeO}(\mathrm{OH})]$.

[Figure 2: X-ray diffraction patterns of metakaolin and red ceramic (RC) used to produce the geopolymers. $Q$ - quartz, $M$ - muscovite, $A$ - analcite, $C$-kaolinite, and $G$-goethite.]

relacionada com a vibração dos grupamentos $\mathrm{OH}^{-}$ligados a átomos de $\mathrm{Al}(\mathrm{Al}-\mathrm{OH})$. Essas ligações podem ser encontradas na caulinita. Essa banda também foi associada com vibrações de estiramento assimétrico do grupo $\mathrm{OH}^{-}$presente nas moléculas de $\mathrm{H}_{2} \mathrm{O}$ fracamente adsorvidas na superfície do material. A banda próxima de $800 \mathrm{~cm}^{-1}$ foi associada com

Tabela III - Composição química das amostras de MK e CV utilizadas para a produção dos geopolímeros.

[Table III - Chemical composition of MK and CV samples use to produce the geopolymers specimens.]

\begin{tabular}{ccccccccc}
\hline Amostra & $\mathrm{SiO}_{2}$ & $\mathrm{Al}_{2} \mathrm{O}_{3}$ & $\mathrm{Fe}_{2} \mathrm{O}_{3}$ & $\mathrm{~K}_{2} \mathrm{O}$ & $\mathrm{CaO}$ & $\mathrm{TiO}_{2}$ & $\mathrm{MgO}$ & $\mathrm{PF}^{*}$ \\
\hline Metacaulim & $54,2 \%$ & $42,5 \%$ & $0,4 \%$ & $0,8 \%$ & $0,1 \%$ & $0,4 \%$ & - & $1,6 \%$ \\
$\mathrm{CV}$ & $57,7 \%$ & $17,0 \%$ & $11,1 \%$ & $5,8 \%$ & $4,5 \%$ & - & $3,9 \%$ & - \\
\hline
\end{tabular}

*-perda ao fogo. 


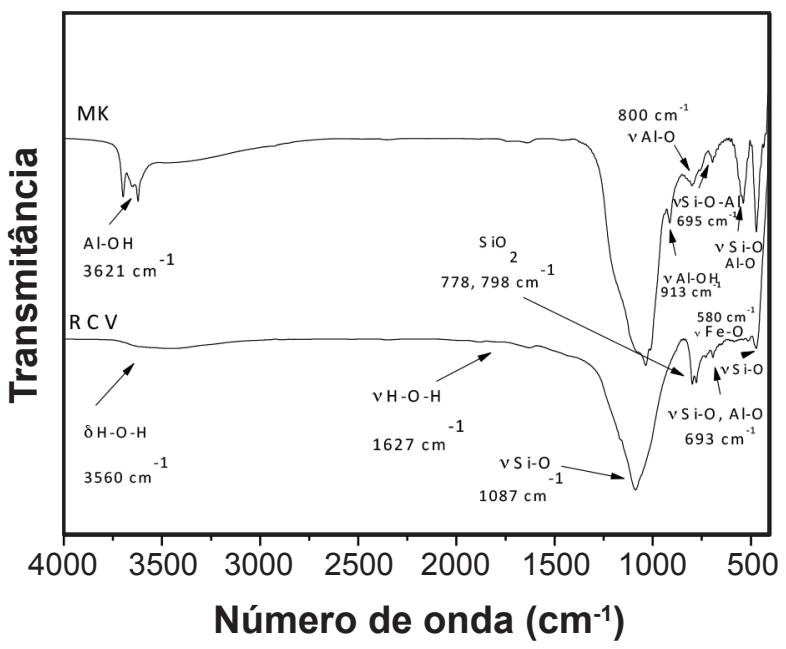

Figura 3: Espectros no infravermelho do metacaulim e da cerâmica vermelha utilizados na produção dos geopolímeros.

[Figure 3: Infrared spectra of metakaolin and red ceramic used to produce the geopolymers.]

vibrações dos grupamentos Al-O e as bandas em $1037 \mathrm{~cm}^{-1}$ foi associada ao estiramento do grupo $\mathrm{Si}-\mathrm{O}$. A banda presente em vibrações próximas de $465 \mathrm{~cm}^{-1}$ foi associada com grupamentos do tipo Al-O-Si presentes em aluminossilicatos. A amostra de CV apresentou uma banda próxima de $3560 \mathrm{~cm}^{-1}$ associada com o estiramento das moléculas de $\mathrm{H}_{2} \mathrm{O}$. Bandas correspondentes ao $\mathrm{SiO}_{2}$ foram observadas em números de onda próximos de 778 e $798 \mathrm{~cm}^{-1}$. Observou-se uma banda em $580 \mathrm{~cm}^{-1}$ referente a vibrações do grupo $\mathrm{Fe}-\mathrm{O}$ presente na goethita. Em $1087 \mathrm{~cm}^{-1}$ foi observada a banda correspondente ao grupamento Si-O. Em $693 \mathrm{~cm}^{-1}$ a banda observada foi relacionada com a presença de grupos Al-O [33].

Microscopia eletrônica de varredura (MEV): as imagens das morfologias dos materiais precursores utilizados na fabricação dos geopolímeros são apresentadas na Fig. 4. As partículas dacerâmica vermelha(Fig.4a) se apresentaram como pequenas partículas não uniformes. Essa não uniformidade pode estar ligada ao processo de moagem promovido. As partículas apresentaram tamanho micrométrico, assim como evidenciado na análise granulométrica (Fig. 1). A imagem referente ao metacaulim (Fig. 4b) demonstrou que as partículas do material se apresentaram mais homogêneas, na forma de aglomerados, e também com dimensões micrométricas, o que comprovou a informação do fabricante (D50= $44 \mu \mathrm{m})$.

\section{Caracterização dos geopolímeros}

Resistência à compressão: a resistência à compressão dos geopolímeros à base de MK e CV após 3, 7, 14 e 28 dias de cura são apresentados na Fig. 5. A ativação do metacaulim promoveu o ganho de resistência mecânica rápido, apresentando uma resistência próxima de $12 \mathrm{MPa}$ após 3 dias de cura [6]. A amostra GPMK-CV após 3 dias de cura apresentou uma resistência à compressão de aproximadamente 9,8 MPa. Um ganho de resistência mecânica próximo de 140\% foi observado para a amostra GPMK após 14 dias de cura. Tais resultados estão de acordo com os resultados encontrados em [34], em que foram obtidas resistências à compressão próximas de $20 \mathrm{MPa}$ após 7 dias de cura dos geopolímeros à base de MK. Esse resultado pode estar relacionado com a maior disponibilidade de aluminossilicato amorfo (presente no MK) capaz de se solubilizar e consequentemente formar as cadeias geopoliméricas responsáveis pelo ganho de resistência mecânica. A substituição parcial do MK pela cerâmica vermelha promoveu a obtenção de matrizes com menores valores de resistência após 14 dias de cura. De acordo com [33], espécies contendo o elemento $\mathrm{Fe}$ quando adicionadas na matriz geopolimérica reprecipitam rapidamente formando hidróxidos ou oxi-hidróxidos, removendo o íon $\mathrm{OH}^{-}$da fase de dissolução. Dessa forma, o processo de dissolução das matérias-primas e a posterior formação dos produtos geopoliméricos é prejudicado.
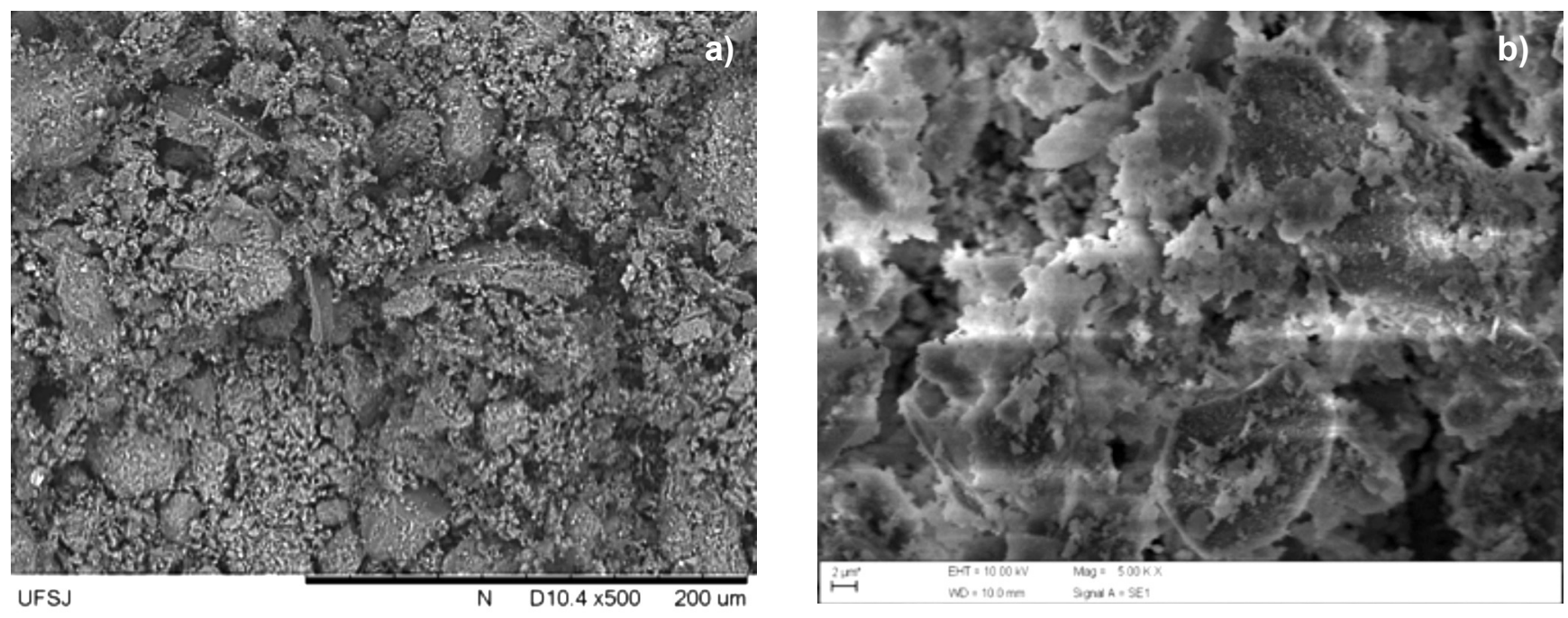

Figura 4: Micrografias obtidas por microscopia eletrônica de varredura das amostras de cerâmica vermelha (a) e metacaulim (b) utilizados como fonte de aluminossilicato nas reações de obtenção dos geopolímeros.

[Figure 4: SEM micrographs of the samples of red ceramic (a) and metakaolin (b) used as sources of aluminosilicate in the reactions for obtaining the geopolymers.] 
Nesse contexto, o ferro ativo presente na $\mathrm{CV}$, mesmo que em baixa concentração, reprecipita na forma de goethita durante a geopolimerização, podendo afetar a dissolução das matérias-primas. Tal processo pode ser relacionado com a diminuição da resistência mecânica observada nas matrizes contendo CV. Além de atuar promovendo a diminuição das espécies $\mathrm{OH}^{-}$no sistema, a adição de $\mathrm{CV}$ dificultou a moldagem dos corpos de prova. Tal dificuldade promoveu o aparecimento de microtrincas e aumento dos valores de porosidade dos CPs, que consequentemente podem interferir de forma negativa no ganho de resistência mecânica dos geopolímeros, como foi observado nos resultados das análises físicas e da morfologia das matrizes enrijecidas. As amostras GPMK-CV e GPMK após 28 dias apresentaram valores de resistência à compressão próximos de 26 e 29 $\mathrm{MPa}$, respectivamente. Ficou evidente que a adição de cerâmica vermelha acarretou na diminuição da velocidade de reação e formação das cadeias poliméricas. Tal fato pode ser observado pelo ganho significativo de resistência da amostra GPMK-CV aos 28 dias. Esses resultados podem estar relacionados com a diminuição do número de espécies $\mathrm{OH}^{-}$no sistema e consequente diminuição na efetividade de dissolução do material precursor. Tais interferências promovem a necessidade de maior tempo para que as reações de geopolimerização e o consequente ganho de resistência mecânica possam ocorrer.

Absorção de água, porosidade e densidade: as propriedades físicas dos geopolímeros obtidos após 3 e 28 dias de cura são apresentados na Tabela IV. A amostra fabricada apenas com MK apresentou valor de absorção de água próximo de $10 \%$ após 3 dias de cura. Após 28 dias de cura o valor de absorção da amostra foi de 5,5\%, demonstrando que o tempo de cura foi fundamental para a obtenção de matriz que absorva menor quantidade de água. O geopolímero teve um aumento nos valores de absorção de água após a adição de CV na matriz. Valores próximos de 36,2 e 37,8\% foram obtidos após 3 e 28 dias de cura, respectivamente. Esse aumento pode estar relacionado com a substituição parcial do metacaulim pela cerâmica vermelha. ACV apresenta uma maior capacidade de absorção de água e menor reatividade quando comparada ao MK. Tal resultado pode ser relacionado ao processo de queima existente na produção de materiais à base de cerâmica vermelha. Os resultados são coerentes com o estudo [28], que demonstra que a inserção de $25 \%$ em massa de materiais contendo concentrações elevadas de ferro promoveu a obtenção de matrizes com 44,5\% de absorção de água após $24 \mathrm{~h}$ de cura. Nesse trabalho, a adição de silicato de sódio durante a produção das matrizes pode ter favorecido a formação de uma maior quantidade de produtos de reação [formação do gel aluminossilicato do tipo N-A-S-H $\left(\mathrm{N}-\mathrm{Na}_{2} \mathrm{O}, \mathrm{A}-\mathrm{Al}_{2} \mathrm{O}_{3}, \mathrm{~S}\right.$ - $\mathrm{SiO}_{2}$ e H - $\mathrm{H}_{2} \mathrm{O}$ )], como apresentado em [35]. Dessa forma, mesmo após a substituição do MK com 50\% em massa de $\mathrm{CV}$, as matrizes apresentaram menores valores de absorção de água quando comparadas aos resultados obtidos em [28].

A porosidade dos corpos de prova sofreu variação quando a cerâmica vermelha foi utilizada como substituinte

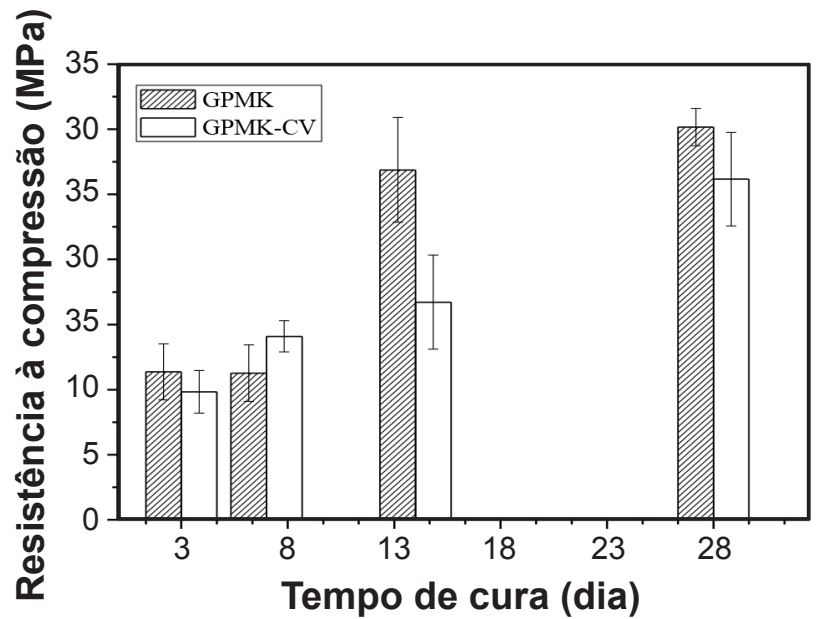

Figura 5: Resistência à compressão dos geopolímeros à base de MK e CV após diferentes dias de cura.

[Figure 5: Compression strength of GPMK and GPMK-RC after different curing times.]

Tabela IV - Absorção de água, porosidade aparente e densidade dos geopolímeros fabricados com metacaulim e cerâmica vermelha após 3 e 28 dias de cura.

[Table IV - Water absorption, apparent porosity and density of metakaolin and red ceramic based geopolymers after 3 and 28 days of curing.]

\begin{tabular}{ccccc}
\hline Amostra & $\begin{array}{c}\text { Tempo } \\
\text { de cura } \\
(\text { dia })\end{array}$ & $\begin{array}{c}\text { Absorção } \\
\text { de água } \\
(\%)\end{array}$ & $\begin{array}{c}\text { Porosidade } \\
\text { aparente } \\
(\%)\end{array}$ & $\begin{array}{c}\text { Densidade } \\
\left(\mathrm{g} / \mathrm{cm}^{3}\right)\end{array}$ \\
\hline \multirow{2}{*}{ GPMK } & 3 & 6,8 & 12,4 & 1,3 \\
& 28 & 5,4 & 10,1 & 1,3 \\
\hline \multirow{2}{*}{ GPMK-CV } & 3 & 36,2 & 46,1 & 2,1 \\
& 28 & 37,8 & 47,3 & 1,9 \\
\hline
\end{tabular}

do MK na produção dos GP. Valor de $12,4 \%$ foi obtido para a amostra GPMK após 3 dias de cura. Após 28 dias a amostra demonstrou uma diminuição na quantidade total de poros presentes, sendo obtido valor próximo de $10 \%$. Após a adição da cerâmica vermelha a matriz experimentou um aumento significativo na porosidade, que pode estar associado com a modificação da taxa reacional de produção dos geopolímeros [33]. As amostras de GPMKCV apresentaram 46,1 e 47,3\% de porosidade após 3 e 28 dias de cura, respectivamente. A substituição do MK em $50 \%$ em massa por cerâmica vermelha promoveu aumentos próximos de 272 e $368 \%$ na quantidade total de poros presentes nas matrizes geopoliméricas após 3 e 28 dias de cura, respectivamente. Tais resultados demonstraram que a substituição parcial do MK pela CV propiciou a formação de uma estrutura mais porosa e que pode interferir na durabilidade das matrizes enrijecidas. A densidade dos corpos de prova fabricados com a adição da $\mathrm{CV}$ foi maior do que a densidade dos corpos de prova das amostras GPMK. Isso pode estar relacionado com a baixa reatividade das partículas de cerâmica que permanecem inalteradas e 
embebidas no gel N-A-S-H formado. Após 28 dias a amostra GPMK-CV apresentou valor de densidade de $1,9 \mathrm{~g} / \mathrm{cm}^{3}$. A amostra GPMK teve densidade de $1,3 \mathrm{~g} / \mathrm{cm}^{3}$ após o mesmo período de cura. Esses resultados demonstraram que, apesar da resistência mecânica das amostras GPMK e GPMK-CV serem similares após 28 dias de cura, as propriedades físicas das amostras apresentaram variações significativas. Tal fato pode ser relacionado com a maior durabilidade das matrizes (menor permeabilidade de água e menor porosidade) e também com a obtenção de matrizes com elevada resistência mecânica e menor densidade, o que é de grande interesse para sua aplicação na indústria da construção civil.

Espectroscopia no infravermelho com transformada de Fourier (IVTF): as Figs. 6a e 6b apresentam os espectros de absorção no infravermelho dos materiais de partida e dos geopolímeros GPMK e GPMK-CV, respectivamente. Em todos os espectros pode-se observar a presença de bandas de absorção em números de onda próximos de $3600 \mathrm{~cm}^{-1}$ e $1600-1700 \mathrm{~cm}^{-1}$ que foram associados com as moléculas de água adsorvidas na superfície do material, como foi também demonstrado em [36, 37]. Essas bandas tendem a aumentar depois do contato do material de partida com as soluções ativadoras [36, 37]. Nos materiais precursores foram observadas bandas próximas de $1080 \mathrm{~cm}^{-1}$ que foram atribuídas ao estiramento dos grupos $\mathrm{Si}-\mathrm{O}-\mathrm{Si}$ presentes nos materiais. Após o contato com a solução ativadora essas bandas se deslocaram para números de onda próximos de $1000 \mathrm{~cm}^{-1}$. Esse deslocamento foi atribuído à substituição de átomos de $\mathrm{Si}$ por átomos de $\mathrm{Al}$ na estrutura geopolimérica, formando, ao término das reações de policondensação, estruturas do tipo Al-O-Si. Essa substituição promoveu a diminuição do comprimento e do ângulo da ligação química após a substituição dos átomos de silício nas estruturas do tipo Si-O-Si por alumínio, formando estruturas do tipo Si-O-Al em que o Si pode ocupar sítios do tipo $\mathrm{Q}^{3}$ ou $\mathrm{Q}^{4}$, dependendo da composição química do sistema, como comentado anteriormente [37, 38]. Esse mesmo deslocamento nas amostras de GPMK-CV ocorreu para frequências mais altas, devido possivelmente à presença de menor concentração do gel do tipo N-A-S-H formado no sistema em função da substituição do MK por CV. Tais resultados estão de acordo com [33], sobre o deslocamento da banda em $1080 \mathrm{~cm}^{-1}$ ser mais pronunciado nas amostras contendo nenhuma ou baixa concentração de $\mathrm{Fe}$ em sua composição. Os autores relacionam o menor deslocamento dessa banda com a menor formação do gel aluminossilicato (geopolímero), o qual é responsável pelo ganho de resistência e a modificação de propriedades físicas, tais como densidade e porosidade. A diminuição da intensidade de bandas próximas de 490$500 \mathrm{~cm}^{-1}$ está associada com a dissolução de espécies de tipo Al-O e Si-O e posterior formação da estrutura Si-O-Al que promove o ganho de resistência mecânica no cimento geopolimérico [33].

As bandas próximas de $1460 \mathrm{~cm}^{-1}$ foram associadas com a formação de espécies carbonatadas $\left(\mathrm{Na}_{2} \mathrm{CO}_{3}\right)[36,37]$. Essas espécies são formadas devido ao excesso de solução alcalina, que reagem com o $\mathrm{CO}_{2}$ atmosférico e formam
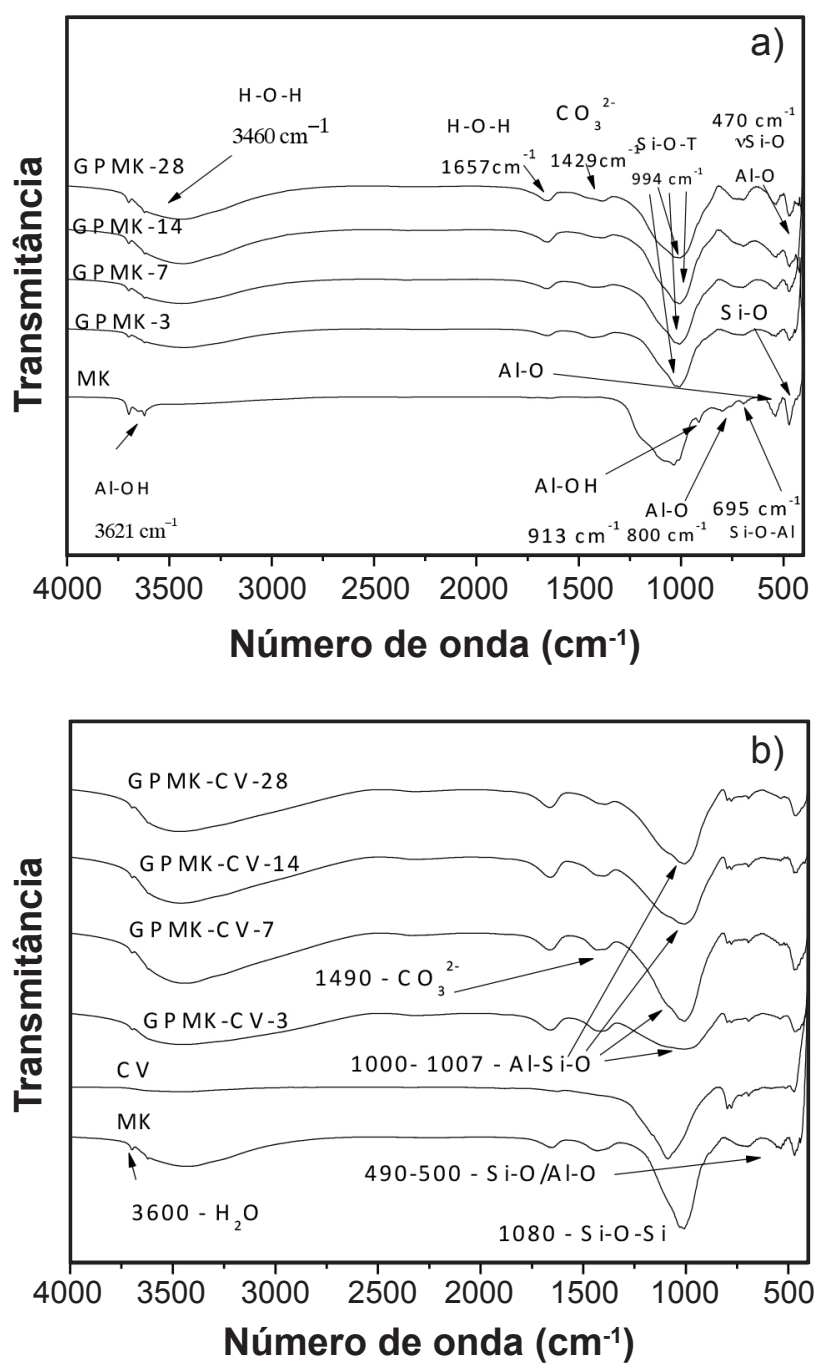

Figura 6: Espectros no infravermelho do MK e dos produtos geopoliméricos (a) e do MK, CV e dos produtos geopoliméricos após diferentes dias de cura (b).

[Figure 6: FTIR spectra of MK and geopolymeric products (a), and $M K, R C$ and geopolymeric products after different curing times (b).]

as espécies identificadas nos espectros de acordo com a equação:

$$
2 \mathrm{NaOH}+\mathrm{CO}_{2} \rightarrow \mathrm{Na}_{2} \mathrm{CO}_{3}+\mathrm{H}_{2} \mathrm{O}
$$

Essas bandas relacionadas com a formação de carbonato de sódio foram mais intensas nas amostras GPMK-CV, principalmente após períodos de cura mais longos (Fig. 6b). Tal fato pode estar relacionado com a maior facilidade do álcali em excesso permear até a superfície dessas amostras, como de fato foi observado nos resultados de porosidade e absorção de água. A baixa reatividade da cerâmica vermelha também pode ser relatada como sendo responsável pelo aparecimento dessas bandas. A solução alcalina que se encontra em maior quantidade em excesso nas amostras GPMK-CV promove a formação de mais $\mathrm{Na}_{2} \mathrm{CO}_{3}$. A substituição do MK (altamente reativo) pelo CV (baixa reatividade) promove reações de eflorescência 
e o aparecimento de bandas relacionadas com $\mathrm{CO}_{3}{ }^{2-}$ nos espectros estudados. Esses resultados também foram relatados no trabalho [39], em que foi utilizada cinza volante ativada com diferentes composições de $\mathrm{Na}_{2} \mathrm{O}$ e $\mathrm{Na}_{2} \mathrm{SiO}_{3}$ e bandas relacionadas com a presença de material carbonatado foram identificadas em amostras após 3,7 e 28 dias de cura.

Difração de raios $X(D R X)$ : as Figs. 7a e $7 \mathrm{~b}$ apresentam os resultados de DRX das amostras GPMK e GPMK$\mathrm{CV}$, respectivamente. É possível observar em todos os difratogramas que os picos referentes às fases de elevado ordenamento atômico continuam presentes mesmo após o contato do material precursor com a solução ativadora. Tal resultado reforça a ideia de que as espécies cristalinas não contribuem (ou contribuem muito pouco) com o processo de dissolução e a consequente liberação de $\mathrm{Si}$ e Al para a formação das cadeias poliméricas. É possível observar que a banda larga correspondente ao material amorfo sofreu um deslocamento. A banda larga, que no material de partida se apresentou entre 18 e $38^{\circ} 2 \theta$, deslocou para angulações próximas de 18 e $43^{\circ} 2 \theta$. De acordo com os trabalhos [17, $34,40]$, esse deslocamento da banda amorfa presente nos
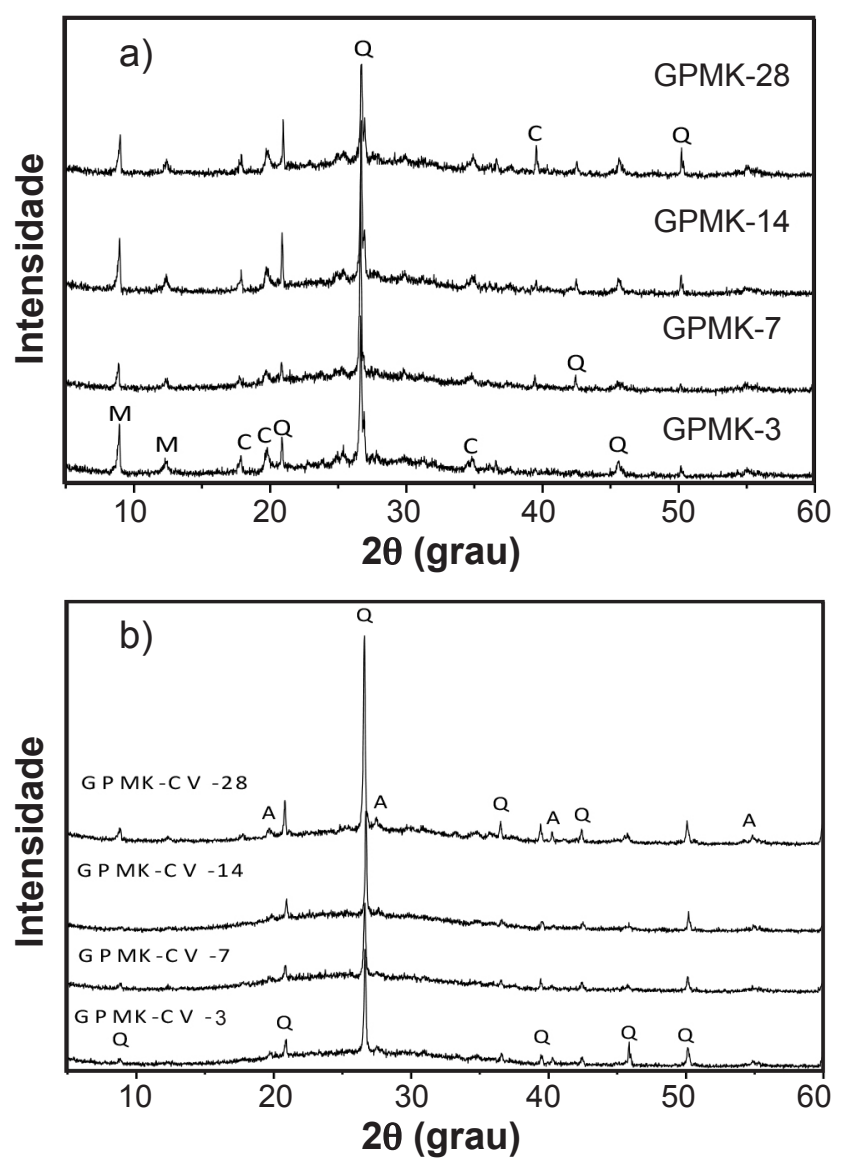

Figura 7: Difratogramas de raios $\mathrm{X}$ dos produtos geopoliméricos GPMK (a) e GPMK-CV (b) após diferentes dias de cura. Q quartzo $\left(\mathrm{SiO}_{2}\right), \mathrm{M}$ - muscovita $\left[\mathrm{KAl}_{2} \mathrm{Si}_{3} \mathrm{AlO}_{10}(\mathrm{OH}, \mathrm{F})_{2}\right], \mathrm{C}$ - caulinita $\left[\mathrm{Al}_{2} \mathrm{Si}_{2} \mathrm{O}_{5}(\mathrm{OH})_{4}\right]$ e A - analcita $\left(\mathrm{NaAlSi}_{2} \mathrm{O}_{6} \cdot \mathrm{H}_{2} \mathrm{O}\right)$.

[Figure 7: X-ray diffraction patterns of GPMK (a) and GPMK-CV (b) geopolymeric products after different curing times. $Q$ quartz, $M$ - muscovite, $C$ - kaolinite, and A - analcite.] produtos geopoliméricos corresponde à formação do gel aluminossilicato (N-A-S-H) com ordenamento atômico de curto alcance, rígido e de elevada resistência mecânica formado após o contato do MK com a solução ativadora. Esse deslocamento pode ser utilizado para observar, conjuntamente ao deslocamento observado nos espectros de infravermelho (1080 para $1000 \mathrm{~cm}^{-1}$ ), a ocorrência da formação dos geopolímeros à base de metacaulim e cerâmica vermelha.

Microscopia eletrônica de varredura (MEV): a Fig. 8 apresenta a morfologia das matrizes geopoliméricas obtidas pela ativação do metacaulim com a solução alcalina após 28 dias de cura. Foi possível observar que a matriz fabricada pela ativação do MK (Fig. 8a) apresentou a formação de um gel aluminossilicato (N-A-S-H), que foi o responsável pelo aumento da resistência mecânica da matriz após o enrijecimento da pasta. Algumas partículas de metacaulim não reagidas estavam presentes juntamente do material formado e se encontraram embebidas pelo gel aluminossilicato formado após a geopolimerização. A presença dessas partículas intactas mesmo após o contato do material precursor com a solução alcalina pode ser associada com a baixa eficiência de dissolução promovida pela solução com a concentração $8 \mathrm{M}$ de $\mathrm{NaOH}$, uma vez que esse parâmetro é de grande importância na produção de polímeros inorgânicos [41]. Foi possível identificar fragmentos de partículas de cerâmica vermelha embebidas no gel formado após a ativação alcalina (Fig. 8b). Devido à substituição de $50 \%$ em massa do metacaulim pela cerâmica vermelha, não foram observadas partículas de MK após o contato com a solução ativadora. A superfície da matriz GPMK-CV se apresentou heterogênea e com a presença de microtrincas. Tais modificações podem estar associadas com a baixa reatividade das partículas de $\mathrm{CV}$ que diminuem a taxa reacional e a consequente formação de uma estrutura mais densificada e homogênea. As partículas de CV se encontraram embebidas do gel aluminossilicato, demonstrando que as partículas, mesmo não participando do

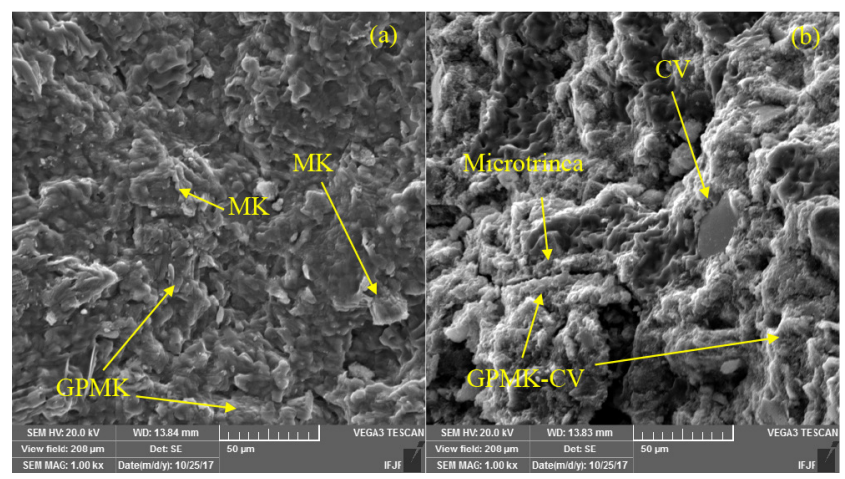

Figura 8: Micrografias obtidas por microscopia eletrônica de varredura das matrizes geopoliméricas produzidas pela ativação do MK com a solução alcalina (a) e com a adição de cerâmica vermelha (b) após 28 dias de cura.

[Figure 8: SEM micrographs of the geopolymeric samples made using MK activated with alkaline solution (a), and with red ceramic (b) after 28 days of curing.] 
processo de dissolução e posterior formação dos polímeros inorgânicos, permitiram a adesão do ligante formado na sua superfície. A presença de defeitos superficiais, tais como microtrincas, pode estar associada com o aumento dos valores de absorção de água e da porosidade aparente das amostras fabricadas com a substituição parcial do MK por cerâmica vermelha.

\section{CONCLUSÕES}

Os geopolímeros produzidos a partir da ativação alcalina do metacaulim (MK) com a solução de hidróxido de sódio e silicato de sódio apresentaram propriedades físicas e mecânicas que favorecem sua utilização em algumas áreas da indústria da construção civil. Os materiais apresentaram um ganho de resistência mecânica dependente do tempo de cura e atingiu os maiores valores aos 28 dias. A substituição parcial do MK pela cerâmica vermelha (CV) não alterou de forma significativa $o$ ganho de resistência à compressão dos corpos de prova. Entretanto, foi possível observar que a adição desse material promoveu a obtenção de matrizes geopoliméricas com maiores valores de porosidade aparente e absorção de água. A pouca reatividade da cerâmica vermelha adicionada na síntese dos geopolímeros promoveu o aumento da densidade dos corpos de prova devido à sua maior densidade quando comparada ao MK. Esse aumento na densidade do ligante inorgânico é uma importante propriedade a ser controlada para sua possível utilização como substituinte do cimento Portland comum em alguns setores da indústria da construção civil. Pelas análises de raios $\mathrm{X}$ foi possível observar que os materiais apresentaram fases com elevado ordenamento atômico, as quais pouco contribuem para a dissolução com a consequente formação do gel aluminossilicato (N-A-S-H). A espectroscopia no infravermelho demonstrou que as reações de geopolimerização ocorreram durante a ativação alcalina mesmo após a adição da cerâmica vermelha. Pelas imagens superficiais dos corpos de prova foi possível observar que a adição de cerâmica vermelha favoreceu a obtenção de matrizes não homogêneas, com a presença de microtrincas e partículas de $\mathrm{CV}$ não reativas que se encontraram embebidas no gel aluminossilicato. Tais resultados demonstraram que a cerâmica vermelha pode ser utilizada como substituinte parcial do metacaulim durante a produção dos geopolímeros. Entretanto, a sua aplicação na indústria da construção civil deve ser tratada com cautela devido às modificações das propriedades físicas das matrizes que necessitam de maiores investigações.

\section{AGRADECIMENTOS}

A Universidade Federal de São João del-Rei (UFSJ), Fundação de Amparo à Pesquisa do Estado de Minas Gerais (FAPEMIG) e Instituto Federal de Educação, Ciência e Tecnologia do Sudeste de Minas - Campus Juiz de Fora.

\section{REFERÊNCIAS}

[1] B.C. McLellan, R.P. Williams, J. Lay, A. Van Riessen, G.D. Corder, J. Clean. Prod. 19, 9-10 (2011) 1080.

[2] D. Hardjito, S.E. Wallah, D.M.J. Sumajouw, B.V. Rangan, ACI Mater. J. 101, 6 (2004) 467.

[3] SNIC, Sind. Nac. Ind. Cimento, "Relatório anual" (2013).

[4] R. Davidovits, C. James, in: Géopolymère ' $99,2^{\text {nd }}$ Int. Conf., Saint-Quentin, France (1999).

[5] J.L.Provis, J.S.J. Van Deventer, Geopolymers: structures, processing, properties and industrial applications, Elsevier (2009).

[6] J.L. Provis, S.A. Bernal, Rev. Mater. Res. 44 (2014) 299.

[7] M.D.B. Barroso, "Desenvolvimento de compósitos com matriz de geopolímeros reforçados com partículas de quasicristais AlCuFe”, Tese Dr., Un. Fed. Paraíba (2009).

[8] A. Palomo, M.W. Grutzeck, M.T. Blanco, Cem. Concr. Res. 29 (1999) 1323.

[9] E.B. Görür, O. Karahan, C. Bilim, S. Ilkentapar, E. Luga, Constr. Build. Mater. 96 (2015) 673.

[10] A. Fernández-Jiménez, A. Palomo, M. Criado, Cem. Concr. Res. 35, 6 (2005) 1204.

[11] Am. Soc. Test. Mater., ASTM C87: "Standard test method for effect of organic impurities in fine aggregate on strength of mortar" (1995).

[12] J.G.S. Van Jaarsveld, J.S.J. Van Deventer, L. Lorenzen, Miner. Eng. 10, 7 (1997) 659.

[13] J. Davidovits, D. Comrie, Geopolymer '88 1 (1988) 125.

[14] F. Škvára, T. Jílek, L. Kopecký, Ceramics - Silikaty 49, 3 (2005) 195.

[15] F. Winnefeld, A. Leemann, M. Lucuk, P. Svoboda, M. Neuroth, Constr. Build. Mater. 24, 6 (2010) 1086.

[16] A.M. Fernandez-Jimenez, A. Palomo, C. LopezHombrados, ACI Mater. J. 103 (2006) 106.

[17] P. Duxson, A. Fernández-Jiménez, J.L. Provis, G.C. Lukey, A. Palomo, J.S.J. Deventer, J.S.J. Van Deventer, J. Mater. Sci. 42, 9 (2006) 2917.

[18] J. Davidovits, Geopolymer '88. 1 (1988) 49.

[19] M.C. Bignozzi, S. Manzi, M.E. Natali, W.D. Rickard, A. Van Riessen, Constr. Build. Mater. 69 (2014) 262.

[20] M. Zhang, T. El-Korchi, G. Zhang, J. Liang, M. Tao, Fuel 134 (2014) 315.

[21] A. Fernández-Jiménez, A. Palomo, Cem. Concr. Res. 35, 10 (2005) 1984.

[22] R.N. Thakur, S. Ghosh, J. Eng. Appl. Sci. 4, 4 (2009) 68.

[23] G. Görhan, G. Kürklü, Compos. Part B Eng. 58 (2014) 371.

[24] P. Kamhangrittirong, P. Suwanvitaya, P. Chindaprasirt, in: $36^{\text {th }}$ Confer. World Concr. Struct., CI-Premier, Singapore (2011).

[25] M.S. Morsy, S.H. Alsayed, Y. Al-Salloum, T. Almusallam, Arabian J. Sci. Eng. 39, 6 (2014) 4333.

[26] E. Arioz, O. Arioz, O.M. Kockar, Procedia Eng. 42 (2012) 1114. 
[27] J. He, J. Zhang, Y. Yu, G. Zhang, Constr. Build. Mater. 30 (2012) 80.

[28] W. Hajjaji, S. Andrejkovičová, C. Zanelli, M. Alshaaer, M. Dondi, J.A. Labrincha, F. Rocha, Mater. Des. 52 (2013) 648.

[29] O.M. Damilola, Syntheses, Int. J. Mater. Sci. Appl. 2, 6 (2013) 185.

[30] P.S. Deb, P. Nath, P.K. Sarker, Mater. Des. 62 (2014) 32. [31] R.Z. Velten, A. Pelissari, S. Ana, D.C. De Lima, C.H. De, C. Silva, C. Alexandre, B. De Carvalho, L. Gontijo, C. Cardoso, Rev. Árvore 30, 2 (2006) 235.

[32] T.W. Cheng, J.P. Chiu, Miner. Eng. 16, 3 (2003) 205.

[33] K. Kaya, S. Soyer-Uzun, Ceram. Int. 42 (2015) 7406.

[34] I. Ozer, S. Soyer Uzun, Ceram. Int. 41 (2015) 10192.

[35] A.G.S. Azevedo, K. Strecker, Ceram. Int. 43 (2017) 9012.
[36] P.N. Lemougna, K. Wang, Q. Tang, X. Cui, Constr. Build. Mater. 131 (2017) 564.

[37] N. Ye, J. Yang, S. Liang, Y. Hu, J. Hu, B. Xião, Q. Huang, Constr. Build. Mater. 111 (2016) 317.

[38] A. Fernández-Jiménez, A. Palomo, Microporous Mesoporous Mater. 86 (2005) 207.

[39] A.G.S. Azevedo, K. Strecker, A.G. Araújo, C.A. Silva, Cerâmica 63, 366 (2017) 143.

[40] J. He, Y. Jie, J. Zhang, Y. Yu, G. Zhang, Cem. Concr. Compos. 37 (2013) 108.

[41] K. Gao, K.L. Lin, D. Wang, C.L. Hwang, H.S. Shiu, Y.M. Chang, T.W. Cheng, Constr. Build. Mater. 53 (2014) 503.

(Rec. 23/04/2017, Rev. 10/08/2017, 16/01/2018, Ac. $03 / 02 / 2018)$ 